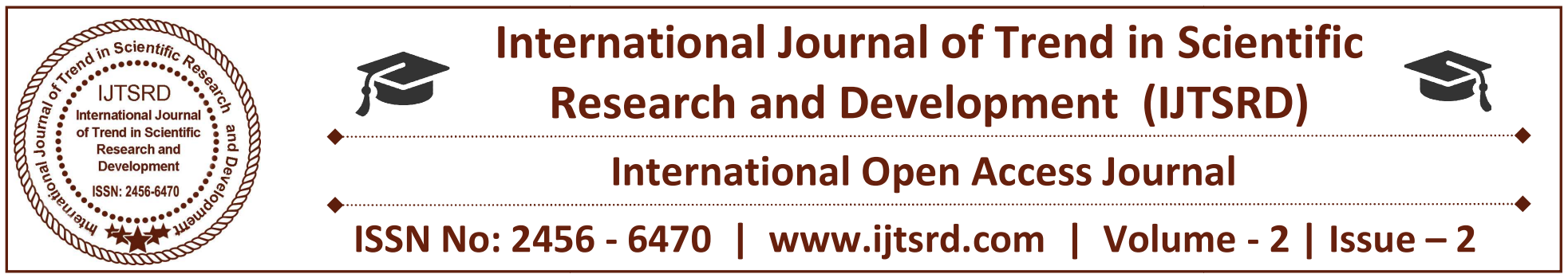

\title{
Human Aspects in Implementation of Total Productive Maintenance
}

\author{
S R Chandra Shashidhar K \\ Research Scholar, Department Of \\ Mechanical Engineering, Industrial \\ Engineering \& Management (Part Time), \\ Institute of Engineering \& Technology \\ (Iet Davv), Indore, Madhya Pradesh
}

\begin{abstract}
Dr. Devendra S Verma
Research Supervisor, Senior Lecturer, Department of Mechanical Engineering, Industrial Engineering \& Management (Part Time), Institute of Engineering \& Technology (Iet Davv), Indore, Madhya Pradesh
\end{abstract}

\section{ABSTRACT}

The manufacturing industry has gone through significant changes in the last decade. Competition has increased dramatically. Customers focus on product quality, product delivery time, safety and cost of product. Because of these, company should introduce a system to improve and increase quality, productivity and safety continuously. The aim of this paper is to study the Human Aspects in Implementation of Total Productive Maintenance. In this paper, we have taken the case study to demonstrate the Total Productive Maintenance implementation methodology and to highlight the benefits achieved during Total Productive Maintenance in an engineering industry XYZ Pvt. Ltd. (name changed) situated in Indore, Madhya Pradesh. TPM proved that its implementation helped the company significantly to achieve higher Productivity, Quality, Cost, Delivery, Safety, Morale, customer satisfaction and profits or the improvements in key performance indicators (PQCDSM). Based on the analysis for achieving operational excellence 5S, LeadTime Reduction / Throughput time Reduction, Kaizen and preventive maintenance are important. Whereas Poka-Yoke and visual control are necessary activities for implementation of Total Productive Maintenance.
Keywords: 5S, Continuous Improvement(Kaizen), Manufacturing Excellence, Lead Time Reduction / Throughput time Reduction, Productivity, Total Productive Maintenance (TPM) ,Human factors, Resistance to change.

\section{Introduction}

$\mathrm{TPM}$ is an innovative Japanese concept. Total Productive Maintenance (TPM) is most important concept for industrial application. The TPM literature offers a number of definitions for Total Productive Maintenance viz. TPM is the general movement on the part of businesses to try to do more With less. (Lawrence 1999 p. 63). TPM is an integrated lifecycle approach to factory maintenance and Support. (Blanchard 1997 p. 72) TPM is a program that "addresses equipment maintenance through a Comprehensive productive-maintenance delivery system covering the Entire life of the equipment and involving all employees from production and maintenance personnel to top management. (McKone, Schroeder etal. 1999 p. 123) TPM is a way of working together to improve equipment effectiveness.(Society of Manufacturing Engineers 1995 p. vii)TPM is a methodology and philosophy of strategic equipment .Management focused on the goal of building product quality by Maximizing equipment 
effectiveness. It embraces the concept of Continuous improvement and total participation by all employees and by all departments. (Society of Manufacturing Engineers 1995 p. ix)TPM is a production-driven improvement methodology that is designed to optimize equipment reliability and ensure efficient management of plant Assets." (Robinson and Ginder 1995 p. 5).

Tpm consists of different methodologies \& pillars.Tpm consists of $5 \mathrm{~s}$ foundation \& 8 pillars namely.

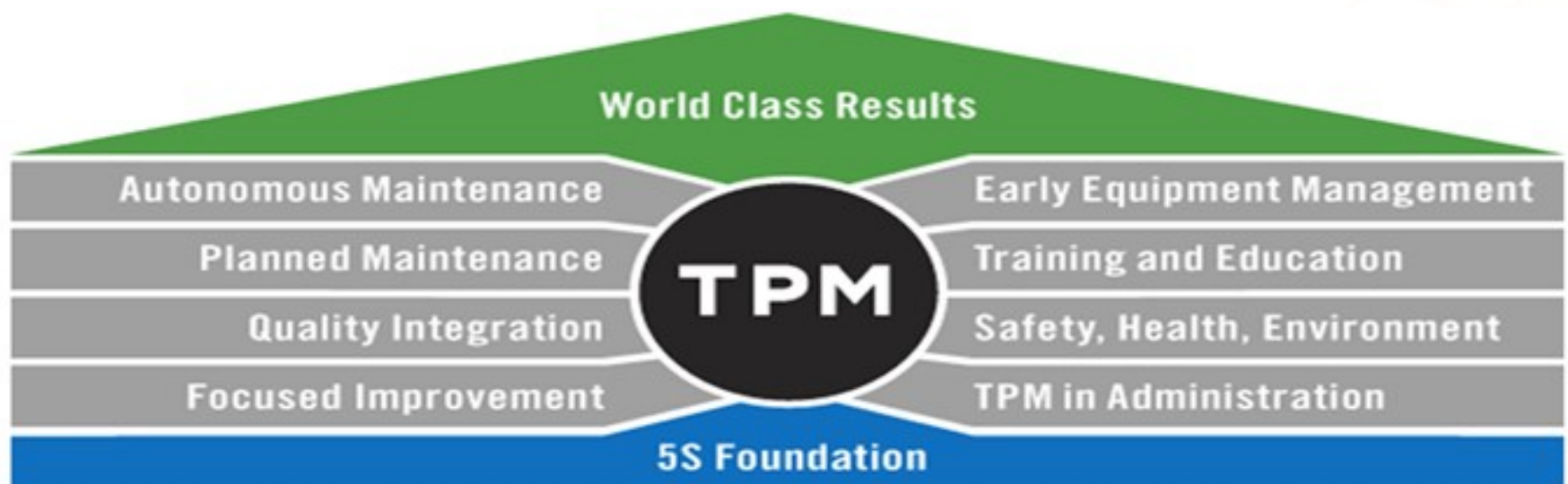

In this paper we will consider implementing the $5 \mathrm{~S}$ to show the human aspects in implementation of TPM.

\section{Implementation Steps}

\section{Deleting non-value added activities through $5 \mathrm{~S}$}

$5 \mathrm{~S}$ practice is a well-recognized key to quality and productivity and for improving the work environment, so it becomes the starting point of TPM programmed. An activity whose removal will not affect a process as it usually generates a zero or negative return of investment on resources is called non-value added activity. The $5 \mathrm{~S}$ system is a lean system used for organizing and managing manufacturing operations so that they require less human effort, space, capital, and time to make products with fewer defects. When 5Ss are properly functioning, they create a visual factory that allows for quick determination of workplace status (Chapman, 2005). "Implementation of 5Ss leads to benefits such as less searching, decreased walking and motion, reduced downtime, fewer safety hazards and accidents, improved flow, fewer mistakes, and better utilization of space" (Chapman, 2005, p.
30). All these benefits result in greater productivity, better quality, less cost, workplace safety, and high morale. 5Ss lays the foundation for the company's overall lean production system, and lack of a robust $5 \mathrm{~S}$ system will make other lean tools ineffective (Chapman, 2005). Implementing 5Ss alone may not bring the desired results, unless itis followed by educating the target work group on the corporate objective and soliciting its support and participation. The failure of most $5 \mathrm{~S}$ programs can beattributed to lack of participation by management in the follow up process (Lewis, 2002). It is essential to have commitment from both top management and others in the organization and to have a $5 \mathrm{~S}$ champion to lead the implementation process (Ho, 1995). The implementation plan is a five step process: first, top management commitment is required; second, a promotional campaign must be run in the organization; third, records should be maintained; fourth, $5 \mathrm{~S}$ training should be carried out; and finally evaluation must be completed. 


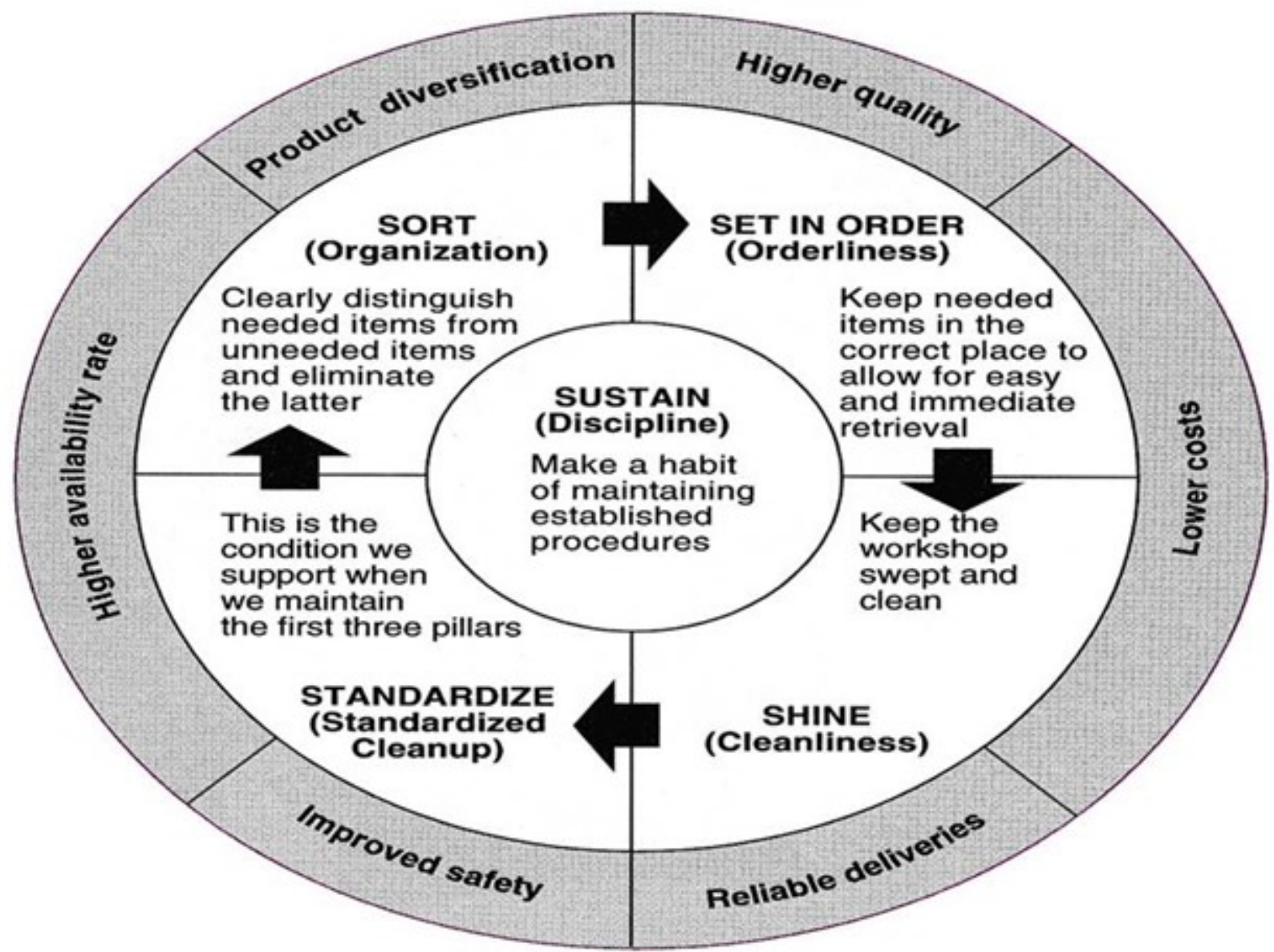

Figure: 5S Representation

\section{Methodology}

\section{Sort}

The first step in $5 \mathrm{~S}$ is sorting. During sorting the team should go through all items in the work area including any tools, supplies, bulk storage parts, etc. The $5 \mathrm{~S}$ team leader should review and evaluate every item with the group. This will help to identify which items are essential for getting the job done effectively and efficiently. If the item is essential for everyday operations it should be tagged and cataloged. If the item is not essential, determine how often it is used in the performance of work in that area. If it is a bulk item, decide the proper amount to be kept in the area and move the remaining quantity to storage. Excess inventory is one form of waste and should be eliminated during the $5 \mathrm{~S}$ activities.

\section{Straighten}

Designate a place for all items that remain in the work area. Put all items in their designated location. An often referenced quote is "A place for everything and everything in its place". During the straighten step, look for ways to reduce or eliminate waste. One form of waste in a process is unnecessary operator motion or movement. Therefore, frequently used tools and supplies should be stored in the immediate work area close to the operator. One effective method commonly used to avoid wasted time searching for the correct tool is constructing shadow boards for all essential tools. Items that are not used as often should be stored based on their frequency of use. All parts bins should be properly labeled. The label should include part number, part description, storage location and the recommended min / max quantities. A properly straightened work area allows the operator to quickly review and verify that they have everything they need to successfully perform their task at hand.

\section{Shine}

The next step is to clean everything in the area and remove any trash. To be effective we must keep the area and any related equipment clean. Dirty process equipment can actually increase the potential for process variability and lead to equipment failure. Lost 
time due to equipment failure is considered waste and non-value-added time. A dirty area can also contribute to safety issues that have the potential to cause a worker to be injured. Operators should clean their areas at the end of each shift. By doing this they will likely notice anything out of the ordinary such as oil or lubricant leaks, worn lift cables, burnt out bulbs, dirty sensors, etc. The purpose is to reduce waste and improve operator safety and efficiency.

\section{Standardize}

The fourth step has been called the most important step in the 5S Process. In this step we must develop the standards for the $5 \mathrm{~S}$ system. They will be the standards by which the previous $5 \mathrm{~S}$ steps are measured and maintained. In this step, work instructions, checklists, standard work and other documentation are developed. Without work instructions or standard work, operators tend to gradually just do things their own way instead of what was determined by the team. The use of visual management is very valuable in this phase. Color coding and standard colors for the surroundings are sometimes used. Photos of the area in the standard $5 \mathrm{~S}$ configuration are often posted for easier identification of non-conformance's. The operators are trained to detect non-conforming conditions and correct them immediately. Schedules should also be developed for regular maintenance activities in each area.

\section{Sustain}

This step in the 5S Process can sometimes become the most challenging of all the five steps. Sustaining is the continuation of the Sort, Straighten, Shine and Standardize steps. It is the most important step in that it addresses the need to perform $5 \mathrm{~S}$ on a consistent and systematic basis. During this step a standard audit system is usually developed and implemented. The goal of the sustain step is to ingrain the $5 \mathrm{~S}$ process into the company culture. The company must strive to make $5 \mathrm{~S}$ a way of life so the benefits gained through the exercise can be maintained. $5 \mathrm{~S}$ is not a one-time exercise. Following the $5 \mathrm{~S}$ Process must become a habit.

\section{Action plan}

Take a picture of the current status of your workplace. Sort to separate anything that is needed and necessary from what is not needed. Organize the things you need so that there is a place for everything and everything has a place. You should be able to find anything in just a few seconds. Clean the workplace and get rid of things that make it difficult to maintain cleanliness, such as boxes on the floor that prevent you from being able to clean the entire surface label them store them in labeled drawers, instead. Prepare an action plan for the items you aren't able to deal with that day, but will be able to in the near future. This could include selling items you no longer use, donating them, recycling, or throwing them away. Take a second picture after the entire day's work, for review.

Prepare Zone Map. Make Zone Plan and Subzones. Identify team for each Zone. Arrange awareness training for $5 \mathrm{~s}$. Kick-off. Implement $5 \mathrm{~s}$. Evaluation of $5 \mathrm{~s}$ implementation in all zones. Documents and display $5 \mathrm{~s}$ results. 


\section{Implementation example}

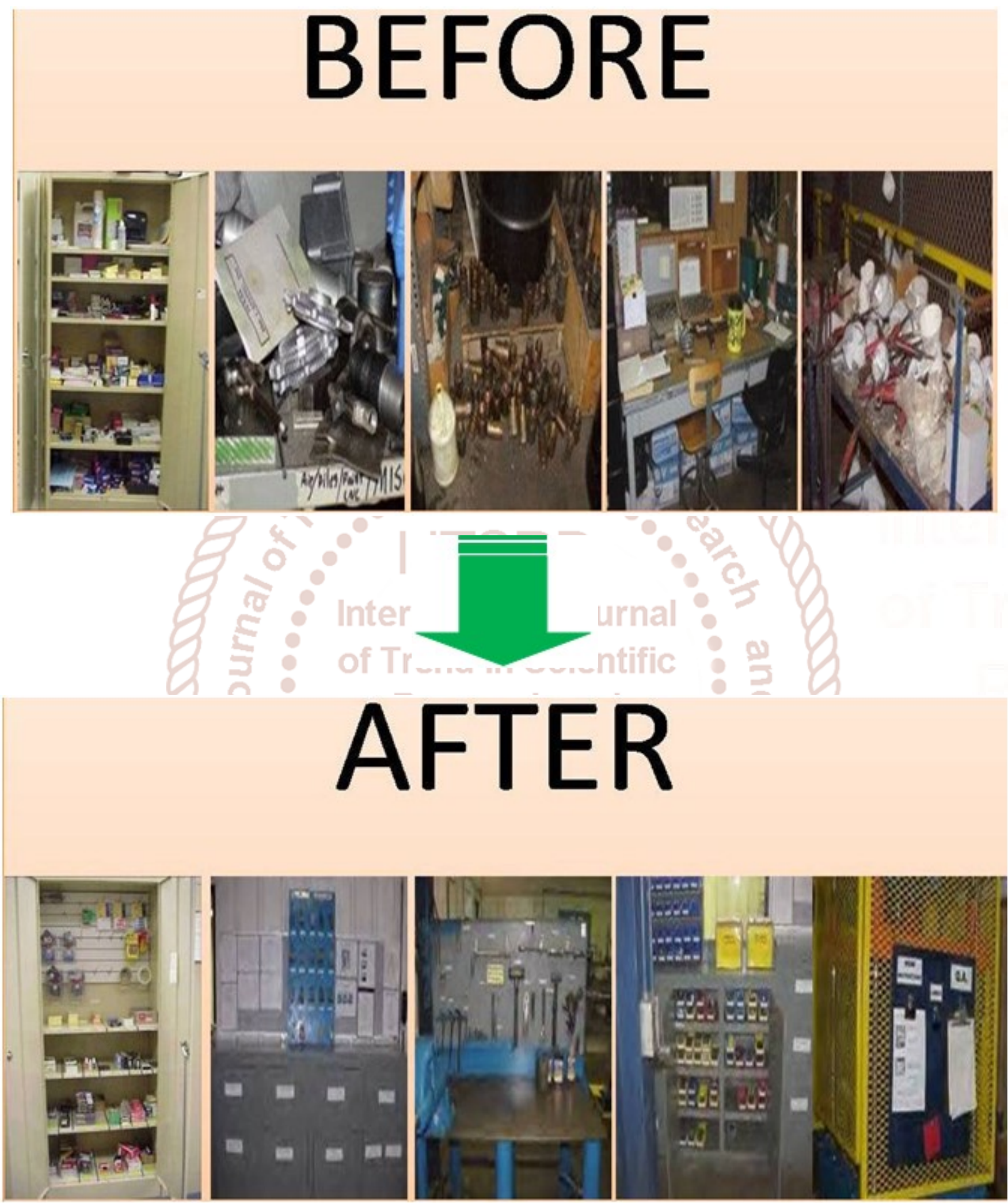

\section{Implementation Summary}

The goal of this work was to get rid of non-value adding operations. Time-wasting and useless movements had been a problem for the company and the 5S-program was an efficient method to break the habit. The results in one area targets were encouraging and gave a good starting point to start expanding the operation model to the rest of the enterprises premises. The project itself kept mostly to the schedule so that duties progressed as planned. Major problems appeared only right at the 
startingphase of the implementation. It was not so clear where to start, because the model was totally new. When things go started the implementing continued quite smoothly.

\section{Problem Statement}

One of the difficulties in implementing TPM as a methodology is that it takes a considerable number of years. The time taken depends on the size of the organization. There is no quick way for implementing TPM. This is contradictory to the traditional management improvement strategies. Following are the other difficulties faced in TPM implementation.
Typically people show strong resistance to change.Many people treat it just another "Program of the month "without paying any focus and also doubt about the effectiveness. Not sufficient resources (people, money, time, etc.) and assistance provided Insufficient understanding of the methodology and philosophy by middle management TPM is not a "quick fix " approach, it involve cultural change to the ways we do things Departmental barrier existing within Business Unit Many people considered TPM activities as additional work/threat.So by using fishbone (Ishikawa) Figure-Fishbone diagram following reasons were found.

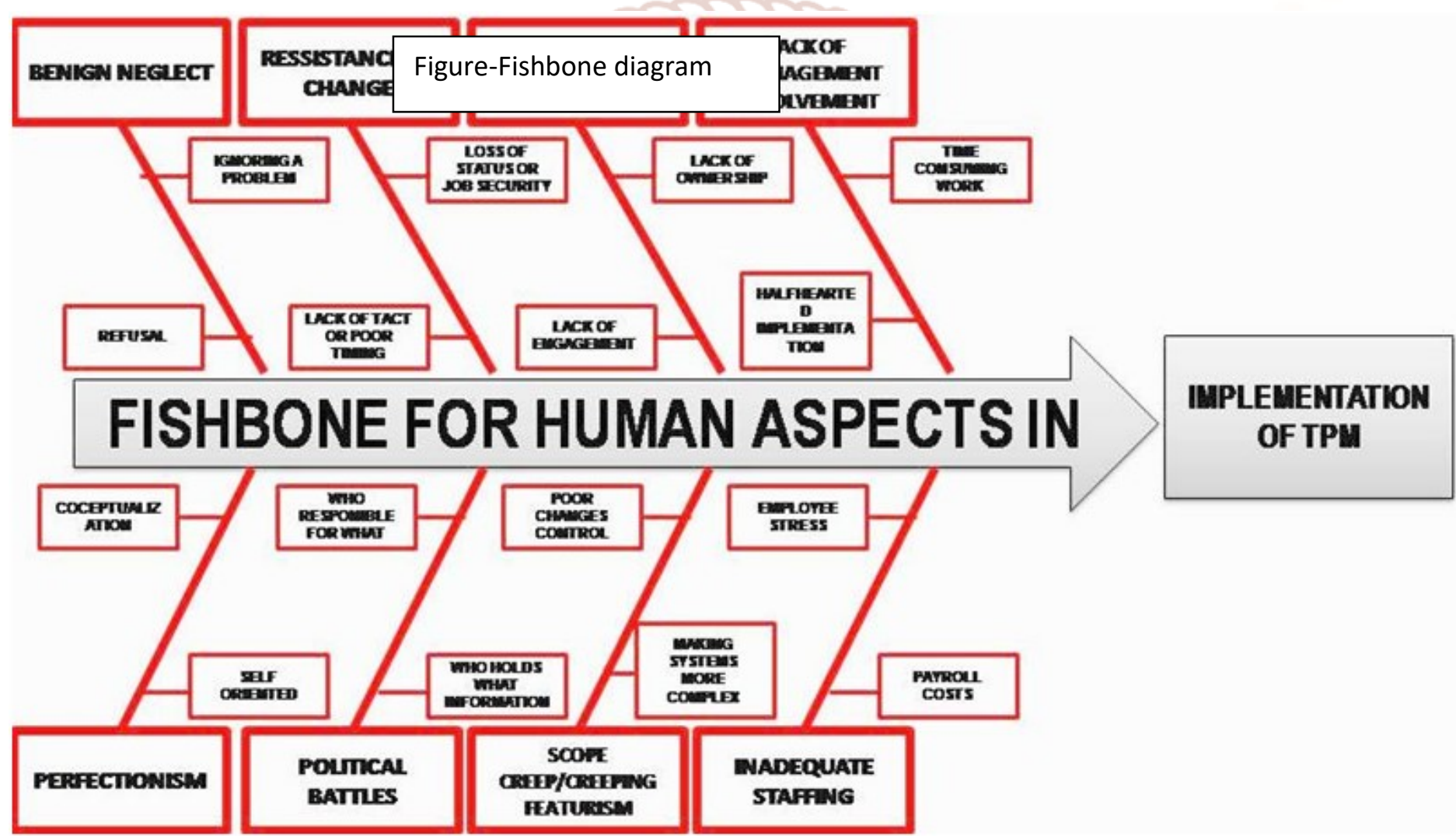

\section{Results \& discussion}

Tpm is a top-down approach and never a down-up approach. Tpm requires an office and not a facilitator. $\mathrm{Tpm}$ is a long term approach not short term. Tpm is all about people and not machines. Tpm is not culture bound.tpm will require a budget. Tpm and company goals must be aligned. management commitment and not support. Tpm is all about people and not machines. Tpm is not culture bound. Management sees Tpm as a cost centre. Lack of tpm knowledge. TPM faces the natural reaction that we all develop against CHANGE. As a matter of fact, any change or alteration in our lives is always a source of concern. TPM represents a change and even though it is always a positive one, employees not always see it that way. For that reason, during the first training sessions we will find resistance, especially from the least educated people. In order to overcome these attitudes, we must create an environment of trust and good will. It is important to create a flow of communication with operators, supervisors, managers etc. When they perceive our sincere purpose of providing them with a better equipment and working environment, most of the barriers will be brought down. Sometimes we will find that the production foremen are not willing to give up their people for the effort because they have a heavy work load. They still think of the maintenance guys taking care of 
everything, the "I break it-you fix it" approach. Maintenance is not the necessary pain or expense any longer. We must see it as a key asset to attain our 21 st. Century goals. It is a Productive Activity.One important step to overcome these challenges is to make sure that people do not see this process just as "Change" but as "Improvement". Everybody wants to Improve. Other valuable concept to keep in mind is to make sure that no one sees this as something "they have to do", but rather as an "opportunity to participate in a successful activity. The successful promotion of the TPM Process and TPM itself involves facing broad challenges and numerous promotion systems with significant responsibilities. Together with enterprise communication, between managers and employees, communication must be improved. Still, companies may confront problems that may make it difficult to focus, to promote longerterm-goals, or less easy to see certain cause \& effects. This is particularly the case when employees' commitment to such activities is not high. To solve these problems, we analyzed the eight pillars, and dissected their contents. This would serve to simplify the four activities for the implementation of TPM.

\section{Conclusion.}

Tpm goals and the company's goals must be aligned, if they are different then you will have a hard time in implementing tpm. Tpm cannot be done by one person or a tpm facilitator, it requires an office, staffed by full-time people with a manager to whom they will report. Managers must be included in pilot machines. Tpm called this the manager's model machine and this is one of requirement. Tpm is costly most specially during the initial stages of the implementation as restoration and correction of abnormalities will take place. Tpm is heavy on documentation, make sure that the people doing it understands this requirement. Documents should not be placed on folders but rather on bulletin boards for everyone to see. Highlight the success, this is the only leverage that you have to sell them to management. Do not present it yourself but rather have the team present them to top management. Tpm will never work through management support, what we need is management commitment \& involvement. Training is very important for tpm start-up activities, all must be trained most specially management. Tpm is a top down approach, and can never work on a down-up approach. The decision to implement tpm must come from the highest person in the industry.

\section{REFRENCES}

1. Tpm Implementation In Small Scale Agriculture Industry: A Case Study Amit Bajaj, Postgraduate Student, G.Z.S.C.E.T. Bhatinda151001, Vipan Kumar,Assistant Professor, Department of Mechanical Engineering, G.T.B.K.I.E.T. Chappianwali, Malout-152107, Punjab, India, International

Journal of Engineering Research \& Technology (IJERT), Vol. 2 Issue 5, May - 2013, ISSN: 22780181

2. Improved organizational behavior through strategictotal productive maintenance implementation. Ahuja, I.P.S., Khamba, J.S. \& Choudhary, R. (2006). PaperNo. IMECE200615783, ASME InternationalMechanical Engineering Congress and Exposition(IMECE), Chicago, IL, November 5-10, 1-8

3. Applying Total Productive Maintenance in Auto Sector: A Case Study with Sona Koyo Group Gurgaon, Sandeep Kumar (Student),Mech.Engg. Department, U.I.E.T, M.D.U, Rohtak, PardeepGahlot (Asst. Professor),Mech.Engg. Department, U.I.E.T, M.D.U, Rohtak, International Journal of Enhanced Research in Science Technology \& Engineering, ISSN: 2319-7463,Vol. 3 Issue 8, August-2014, pp: (271-275), Impact Factor: 1.252

4. TPM implementation to invigorate manufacturing performance: an Indian industrial rubric Murugadoss $\mathrm{K}$ Panneerselvam, International Journal of Scientific\& Engineering Research, Volume 3, Issue 6, June-2012 1 ISSN 2229-5518 IJSER (C) 2012 http://www .ijser.org

5. Total productive maintenance, Handbook of Maintenance Management and Engineering, Ahuja, P. 2009.

6. Relationship of $5 \mathrm{~S}$ and Manufacturing Performance with Mediator of TPM and TQM B. DevarajNaik, LakhanPatidar, Pradeep Kumar Soni, International Research Journal of Engineering and Technology (IRJET) e-ISSN: 2395 -0056 Volume: 02 Issue: 07 | Oct-2015 www.irjet.net p-ISSN: 2395-0072 C 2015

7. The impact of total productive maintenance practices on manufacturing performance. Kathleen E. McKone a; Roger G. Schroeder b, Kristy O. Cuab(2015) 
8. Total Productive Maintenance: Need \& Framework, Yash Parikh, PranavMahamuni (2015), ISSN: 2349-2163, Issue 2, Vol. 2

9. Relationship of $5 \mathrm{~S}$ and Manufacturing Performance with Mediator of TPM and TQM, B. DevarajNaik, LakhanPatidar, Pradeep Kumar Soni (2015), e-ISSN:2395 -0056 Vol: 02 Issue: 07, p-ISSN: 2395-0072

10. Review Paper On: Total Productive Maintenance, Narinder Singh, Onkar Singh Bhatia, (2014), ISSN: 2454-132X

11. TPM-A PDCA Approach, P.K.Suresh, Dr.M. Joseph, Dr.J.Raj. (2014) [12] A review of tangible benefits of TPM implementation, Arunraj K., Maran M (2014), ISSN 2277 - 9442, Vol. 3, Issue 1

12. Review Paper on TPM- A Key Strategy for productivity Improvement in Medium Scale Industry, Wasim.S.Hangad, Dr.SanjayKumar(2013), ISSN 2229-5518 Vol.4, Issue 11

13. Investigation of Human Aspect ein Total Productive Maintenance (TPM). A Methodology for Implementing Total of Productive Maintenance in Manufacturing Industries-A Case Study Total Productive Maintenance: A Case Study in Manufacturing Industry.
14. The Pathway to Operational Excellence in the Pharmaceutical Industry - Overcoming the Internal Inertia.

15. TPM orients enterprises towards production excellence: A Practical Analysis of OEE. Liu Yong, Zhu Jiajia.

16. Total productive maintenance: Literature review and directions, International Journal of Quality and Reliability Management, Ahuja I.P.S., Khamba, J.S., (2008), Vol.10 No.2, pp. 93-106

17. Total Productive Maintenance: A Case Study in Manufacturing Industry, M.WorknehWakjira, Ajit Pal Singh (2012), Vol.25 No.1, ver. 01

18. Factors affecting successful implementation of total productive maintenance:a UK manufacturing case study, Journal of Quality in Maintenance Engineering, Bamber,C.J.,Sharp,J.M. and Hides,M.T. (1999), Vol. 2 No.3, pp 4-20

19. Upgrading Operational Capabilities with the Implementation of TPM Initiatives in an Indian Industry: A Case Study, Pardeep Gupta (2015), ISBN: 978-1-63248-074-3

20. An enhanced approach for implementing total productive maintenance in the manufacturing environment, Journal of Quality in Maintenance Engineering, Blanchard, B.S (1997), Vol. 1 No. 1, 1995, pp. 20-26 